\title{
Numerical Study on Flow Characteristics of A Micro Jet Actuator
}

\author{
Bo-fu $\mathrm{Wu}^{1, *}$, Shi-wei $\mathrm{Li}^{2,3}$ \\ ${ }^{1}$ School of Automotive and Traffic Engineering, Hefei University of Technology, \\ Hefei, 230009, China \\ ${ }^{2}$ School of Aeronautics, Northwestern Polytechnical University, Xi'an, 710072, China \\ ${ }^{3}$ Low Speed Aerodynamics Research Institute, China Aerodynamics Research and Development \\ Center, Sichuan mianyang 621000, China
}

Keywords: Jet Actuator, CFD, Inlet Pressure

\begin{abstract}
In order to reduce fuel consumption and vehicle exhaust emissions, it is necessary to develop new designs, such as flow control using jet actuators, to reduce vehicle aerodynamic drag. A numerical model was developed based on the process of airflow passed through a micro-jet actuator. Under different inlet pressure, the flow field and its characteristics were analyzed with computational fluid dynamics (CFD) method. The numerical results show that a high outlet airflow velocity can be obtained as the inlet pressure exceeds $1.0 \mathrm{e} 5 \mathrm{~Pa}$ for this actuator. Therefore, this type of actuator can be used for vehicle airflow control to reduce aerodynamic drag.
\end{abstract}

\section{Introduction}

Nowadays, the constant need for better fuel economy and greater vehicle performance prompt automobile developers to reduce aerodynamic drag with different ways. The vehicle aerodynamic drag comes mainly from the wake flow separation at the rear area of the vehicle body. Therefore, keeping the separated flow attached over as much of the rear body surface as possible is an effective way to reduce vehicle aerodynamic drag [1]. Recent advances applied in aeronautical applications shows great prospects to control the separated flow of vehicles with micro-jet actuator [2].

Because the aerodynamic drag of vehicle is strongly related to the flow downstream of the vehicle, blowing high speed airflow with micro-jet actuator located slightly upstream of the detached wake flow has to be proved as an successful way to reduce aerodynamic drag [3]. Up to now, many works have been carried out to investigate the performance of micro-jet actuators. An experiment of flow control for a 3D bluff body using micro-electro mechanical system was investigated [4]. The results showed that a significant aerodynamic drag was obtained with micro-jets located upstream the recirculation bubble, and the energy balance of using micro-jet actuator was evaluated in compared with conventional magnetic valves. The first experiment of the efficiency of micro-jets to control the detached airflow on a full-scale production car was performed, which demonstrated the feasibility of the controlling the separated airflow to reduce the aerodynamic drag [5]. Ducloux et al. designed and fabricated a new micro-jets based on the induction of a strong coupling between a mobile mechanical part and a high pressured airflow [3]. These works built a solid basis of designing new type micro-jets, and shows great perspective of future applications of flow control in vehicle industry.

The aim of the present study is to investigate the airflow characteristics inside an actuator designed by Ducloux et al. In order to describe the process of airflow passed through the micro-jet actuator, a numerical model was developed with CFD model. With simulations, the blowing high speed airflow process for the micro-jet actuator was investigated using CFD program Ansys 17. 0. The effects of different inlet pressures on the characteristics of the micro-jet actuator were analyzed in a systematic manner, and an optimum input pressure was recommended. 


\section{Numerical Model}

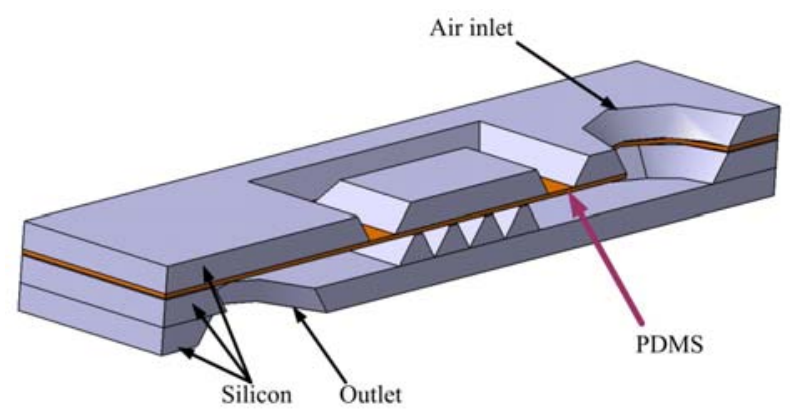

Fig.1 Simplified representation of the actuator.

Fig.1 shows a Micro-electromechanical Systems (MEMS) actuator designed by Duclox [3]. The schemes and fabricated process are detailed in Duclox, et al. [3,6]. The micro-jet actuator is the most important component affecting the flow control performance of reducing aerodynamic drag. As shown in Fig.1, as constant high pressure airflow at the air inlet flows through the channel consisted of silicon and polydimethylsiloxane (PDMS) membrane, the PDMS membrane will vibrate under the inlet pressure. Thus, high frequency airflow blows from the outlet, which can be used in separated flow control to reduce aerodynamic drag. The velocity of airflow blowing out from the outlet can be adjusted by changing the inlet pressure level. In this study, the channel fabricated by silicon and PDMS are chosen as the research object for a more effective analysis, and the physical model and its main dimensions (pressure inlet $=1.5 \mathrm{e} 5 \mathrm{~Pa}$ ) are shown in Fig.2.

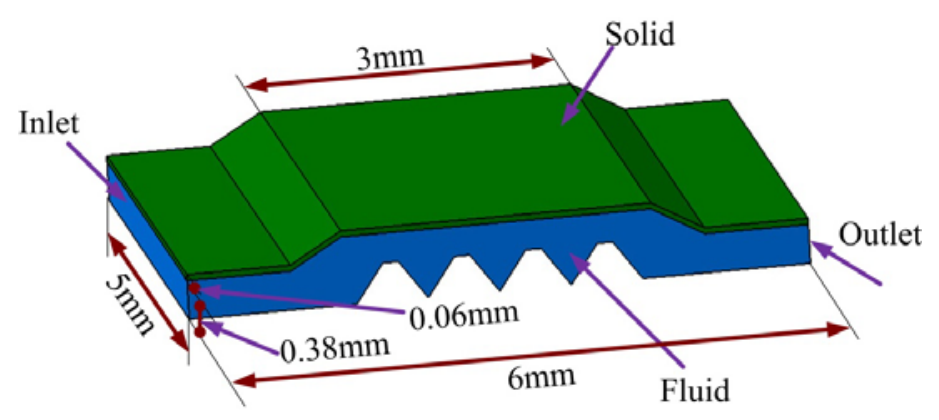

Fig.2 Phycical model of the actuator (pressure inlet $=1.5 \mathrm{e} 5 \mathrm{~Pa}$ ).

The airflow passing through the actuator was considered unsteady and incompressible, the Reynolds-Averaged Navier-Stokes equations for conservation of mass and momentum can be written as

$$
\begin{aligned}
& \frac{\partial \rho}{\partial t}+\frac{\partial u_{i}}{\partial x_{i}}=0 \\
& \frac{\partial\left(\rho u_{i}\right)}{\partial t}+\frac{\partial}{\partial x_{j}}\left(\rho u_{i} u_{j}\right)=-\frac{\partial P}{\partial x_{i}}+\frac{\partial}{\partial x_{j}}\left(\mu \frac{\partial u_{i}}{\partial x_{j}}+\tau_{i j}\right)+\rho g_{i}+\tau_{f}
\end{aligned}
$$

where $u_{i}$ is the airflow velocity along the coordinate ${ }^{x_{i}} ; \rho$ is the air density; $P$ is the pressure; $\mu$ is the viscosity; and $\tau_{i j}=-\rho \overline{u_{i}^{\prime} u_{j}^{\prime}}$ is the Reynolds stress, which represents the effects of turbulent fluctuation.

The conservation of solid membrane can be given as

$$
\rho_{s} \ddot{d}_{s}=\nabla \cdot \sigma_{s}+f_{s}
$$


where $\rho_{s}$ is the solid membrance density, $\sigma_{s}$ is the Cauchy's stess tensor, $f_{s}$ is the volume force, $d_{s}$ is the solid menbrance local acceleration.

At the solid-fluid interface, the coupling system should satisfy that fluid displacement and solid displacement are equal, and the conservation of both stress are equal. The airflow passing through the actuator is turbulent flow due to its high velocity, and the $k-\varepsilon$ turbulent model was selected to close the governing equations.

The equations described above were solved numerically by using finite volume based Ansys 17.0 code. The boundary condition for airflow at the inlet was set up as pressure inlet with a value of 1.0e5 $\sim 1.5 \mathrm{e} 5 \mathrm{~Pa}$ and the outlet was treated as standard atmosphere condition. At the walls, no slip boundary condition was applied for velocity, and a standard wall function was utilized for the near wall treatment.

\section{Results and Discussion}

Velocity and turbulent intensity are two important indicators to understand the flow field of the actuator. Under different inlet pressure, the airflow velocity distribution and the contours of turbulent intensity at the actuator symmetry plane are presented in Figs. 3 and 4, respectively.

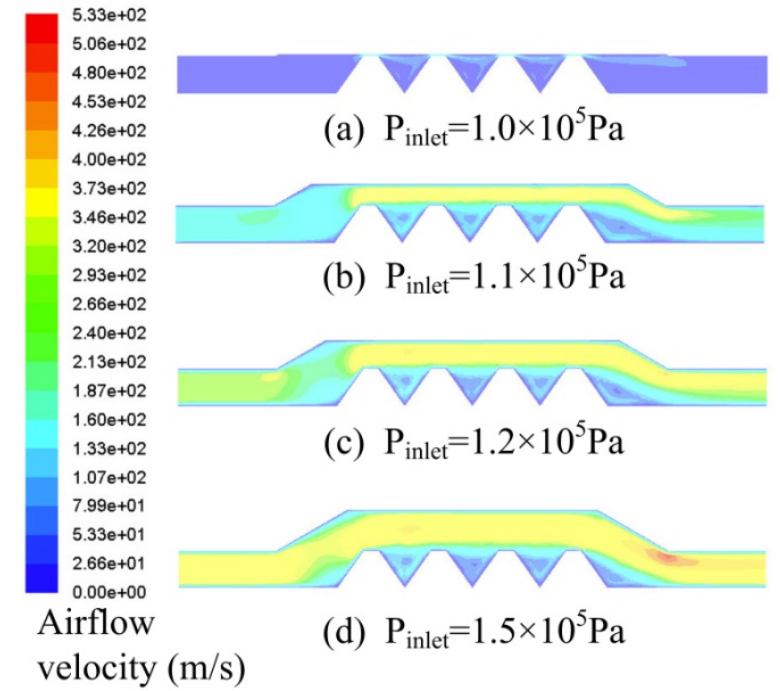

Fig.3 Airflow velocity distribution at actuator symmetry plane.

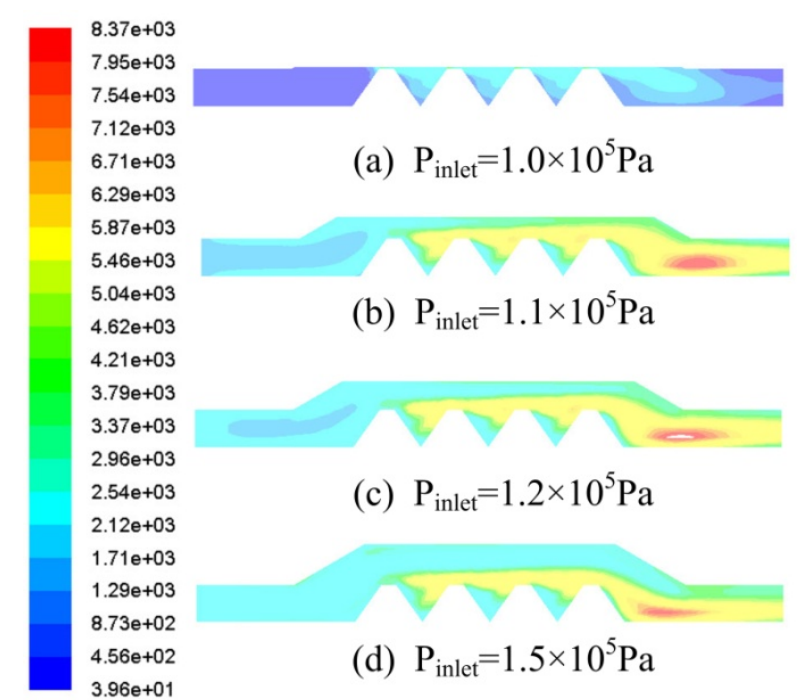

Fig.4 Contours of turbulent intensity at actuator symmetry plane.

From Fig. 3, it can be seen that the inlet pressure has great effects on the airflow velocity distribution of the actuator. The height of the flow path increases with the increasing the input pressure. As shown from Fig. 3(a), the vibration between the fluid and PDMS is very small. The main reason is that the pressure inlet is not large to open the clearance between the solid structures. Thus the airflow velocity is much smaller than that of higher inlet pressure. It can be seen clearly that, as inlet pressure increases from $1.1 \mathrm{e} 5 \mathrm{~Pa}$ to $1.5 \mathrm{e} 5 \mathrm{~Pa}$, the coupling effect between the passing airflow and the PDMS membrane becomes stronger.

Fig. 4 shows the turbulent intensity at the actuator symmetry plane under different inlet pressure. As expected, the size of the high turbulent intensity region increases with the increase of inlet pressure, which is helpful for the vibration of the PDMS membrane. From Figs. 3 and 4, it can be seen that in order to obtain a high outlet airflow velocity, a certain amount of inlet pressure is required. 


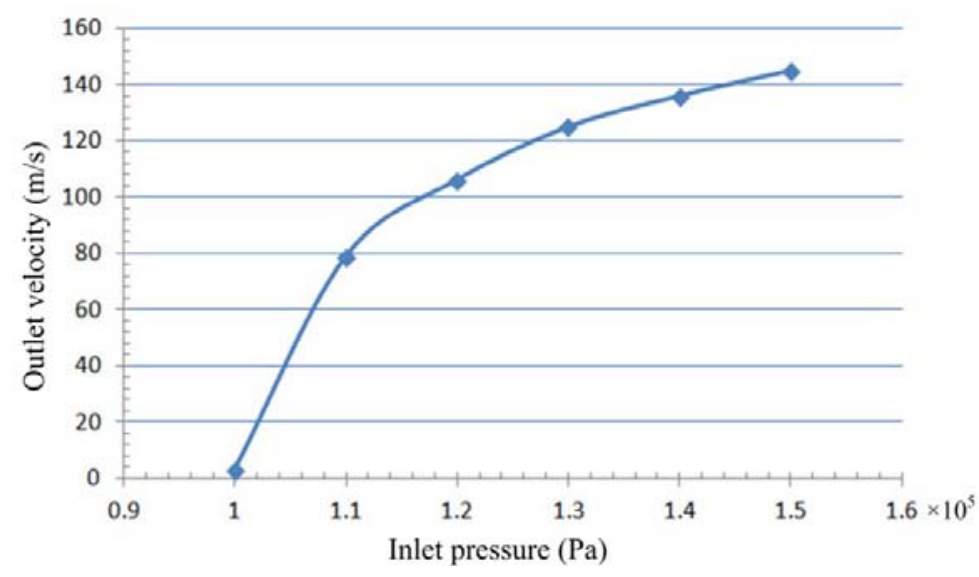

Fig. 5 Effects of inlet pressure on outlet airflow velocity.

Fig. 5 shows the average velocity of airflow blowing out from the outlet as a funciton of inlet pressure. Airflow velocity plays a important role in evaluating performance of actuator. As shown in Fig. 5, the outlet airflow velocity increases with the increasing of inlet pressure. This can be expected that a higher inlet pressure supply more energy than that of a lower inlet pressure. From Fig. 5, it can be concluded that the input pressure correspond to the number of $1.0 \mathrm{e} 5 \mathrm{~Pa}$ is not enough because the outlet airflow velocity is $2.87 \mathrm{~m} / \mathrm{s}$, which is too low to control airflow separation for reducing aerodynamic drag. For this actuator, higher inlet pressure can result in higher outlet airflow velocity, but the effect of the inlet pressure on outflow velocity begins to decline when increasing inlet pressure from $1.4 \mathrm{e} 5 \mathrm{~Pa}$ to $1.5 \mathrm{e} 5 \mathrm{~Pa}$. From the point of view of trade-off, the inlet pressure of $1.4 \mathrm{e} 5 \mathrm{~Pa}$ is an optimal operating point for this actuator.

\section{Conclusions}

This study is deal with the numerical investigation of a micro-jet actuator. With simulations, the airflow characteristics and outlet airflow velocity of the actuator were evaluated for different inlet pressure. It is observed from the simulation results that the minimum inlet pressure should exceed $1.0 \mathrm{e} 5 \mathrm{~Pa}$. Higher inlet pressure means high energy consumed, and the inlet pressure of $1.4 \mathrm{e} 5 \mathrm{~Pa}$ is recommended from the point of view of energy trade-off.

\section{Acknowledgements}

The financial support of State Key Laboratory of Aerodynamics Open Project (SKLA2015h0201) is gratefully acknowledged.

\section{References}

[1] P. Bearman, The Aerodynamics of Heavy Vehicles II: Trucks, Buses, and Trains, Springer (2009) 3-13.

[2] D. Greenblatt and Israel J. Wygnanski, Progress in Aerospace Science. 36(2000) 487-545.

[3] O. Ducloux, A. Talbi, L. Gimeno, R. Viard and P. Pernod, Applied Physics Letters. 91(2007) $1-3$.

[4] J. Pierric, A. Xavier, E. Christophe and A. Jean-Luc, Experiments in Fluids. 54(2013) 1-12.

[5] A. Jean-Luc, J. Pierric, R. Tony, G. Philippe, E. Yoann, E. Christophe and A. Xavier, International Journal of Flow Control. 6(2014) 1-20.

[6] O. Ducloux, Y. Deblock, A. Talbi, L. Gimeno, N. Tiercelin, P. Pernod, zV. Preobrazhensky and A. Merlen, Solid Mechanics and its Applications, IUTAM Symposium on Flow Control and MEMS Proceedings of the IUTAM Symposium. 7(2008) 59-75. 